\title{
Interferometers based on standing wave detection using thin film photodiodes
}

\author{
Non-member \\ Member \\ Xiaoyu Mi (Tohoku University) \\ Non-member \\ Minoru Sasaki (Tohoku University) \\ Member \\ Takayuki Hirano (Tohoku University) \\ Kazuhiro Hane (Tohoku University)
}

\begin{abstract}
Novel optical interferometers based on detecting a standing wave are realized with a newly developed ultra-thin film photodiode. The active layer of the ultra-thin film photodiode is thinner than half of the wavelength of the incident light. Only a small percentage of the incident photons are absorbed and the rest pass through the photodiode. Since the photo-sensitive layer of the photodiode is thinner than the pitch of an optical standing wave, which is the thinnest interference fringe generated by two counter-propagating beams, the standing wave is measured by inserting the ultra-thin film photodiode in the optical field. Taking advantage of this function, two kinds of compact interferometers are realized. One requires the mirror on the sample surface and the other can use the rough surface. The sensor package can be small not using the wave-guide. The principle, design, fabrication process, and performance as a displacement sensor are described.
\end{abstract}

Keywords: interferometry, ultra-thin film photodiode, standing wave, speckle, displacement sensor, integration

\section{Introduction}

Laser interferometry is a well-developed technique for displacement measurement since it has high resolution and gives noncontact method ${ }^{(1)}$. In most interferometers, a half mirror is used for making sensing and reference beams from one laser beam and for superposing these bearns after their travels through interferometer arms.

Compact optical interferometric sensors are advantageous for installing in many industrial equipments because of their advantages of small mass and size, stability, no alignment adjustment, and probably the low cost. Application flexibility will be greatly improved. Although there is a great demand for integrated displacement sensors, the practical displacement sensor is still poor in signal amplitude, contrast, and the dynamic range of the measurable distance. Only in the expensive and huge equipments (e.g., stepper, electron beam drawer), the bulky interferometer is used.

The approach to building integrated interferometers has been based on the miniaturization of two-beam interferometers using the wave-guide on a plane surface ${ }^{(2)(3)}$. As a compact interferometric sensor, the optical feedback technique has been studied ${ }^{(4-6)}$. The basis is the mixing between the back reflected sensing beam from a moving sample and the optical field inside the laser diode. This technique gives very simple optical configuration, since only one optical arm is needed, its optical configuration is very simple. The dynamic range of the measurement, however, is limited by the mode hopping of the laser diode and the lack of stable operation.

Recently, we have proposed a compact interferometer based on standing wave detection using a thin film photodiode ${ }^{(7)}$. The key device is an ultra-thin film photodiode. The optical configuration is as simple as that of the optical feedback technique. When a coherent light beam is normally incident on a reflection mirror, the standing wave occurs in front of the reflection mirror. The use of an ultra-thin film photodiode to detect the standing wave eliminates the need for a beam spliter and the optical arm for reference whereas the usual two-beam interferometer requires two arms. The interferometer is essentially stable having the promise of integration and suitable for a small sensor. This advantage is still valid, even when the reflection becomes a speckle field due to the rough surface. For some industrial applications, the object frequently has the rough surface causing random scattering. A speckle interferometer has been already developed for this requirement ${ }^{(8)}$. A kind of moire fringe is generated between a pair of the speckle patterns obtained before and after the object displacement. Statistical image processing, however, is usually necessary to extract the displacement. The proposed interferometer uses a thin film photodiode to detect the standing wave inside a speckle pattern as far as the signal magnitude is large enough.

In this paper, two kinds of interferometers based on standing wave detection are described for developing a compact interferometric displacement sensor. The properties of these interferomters are investigated. 
(a)

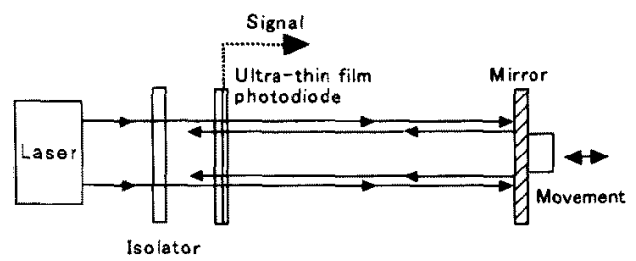

(b)

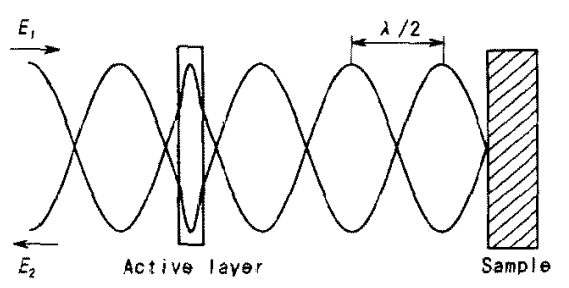

Fig. 1(a). Interferometer using an ultra-thin film photodiode. (b). Schematic diagram to explain the principle of standing wave detection.

\section{Principle and designing}

A schematic diagram of the proposed interferometer based on standing wave detection using a thin film photodiode is shown in Fig.1. The interferometer consists of a laser (including collimation lens), an isolator, and a newly developed thin film photodiode. The reflection mirror is on the moving object. The active layer of the ultra-thin film photodiode which absorbs photons is designed to transmit most of the incident light beam. In our design, the absorption ratio to the incident light power is estimated to be less than $1 \%$. The almost incident light beam transmits through the photodiode before being absorbed, and travels to the mirror on the moving object and returns to the thin film photodiode. The incoming and reflection light beams superpose each other and produce a standing wave which penetrates the active layer. The period of the standing wave is $\lambda$ $/ 2 n$ ( $\lambda$ is the wavelength of the incident light, $n$ is the refractive index of the media). Having a node on the reflection mirror, the intensity profile of the standing wave is spatially fixed to the reflection mirror. If the active layer has the appropriate thickness, which is at least thinner than the period of the standing wave of $\lambda / 2 n$, the intensity profile of the standing wave can be resolved. When the photodiode is at the node of the standing wave, the signal decreased, and when the photodiode is at the anti-node, the signal increases. The relative position between the ultra-thin film photodiode and the mirror decides the photodiode signal. This interference signal gives the displacement between the ultra-thin film photodiode and the moving sample using the standing wave as the standard rule. Neither a reference mirror nor beam splitter is necessary in this interferometer. The wavefront of the incoming laser beam is the reference. Since a laser beam having a large diameter can be used inside the sensor system, beam expansion due to the diffraction is small. The measurement of the long distance up to the coherent length of the light source will be possible. In addition, a dual photodiode integrated with a phase shifter is fabricated to detect the displacement direction. A phase difference of $\pi / 2$ can be introduced between two interference

signals obtained from two photodiodes by inserting a phase shifter in front of one of the two photodiodes(see Fig. 3.). By comparing the phases of two signals, the displacement direction can be determined.

The detail of the mathematical expression for the standing wave signal is described elsewhere ${ }^{(7)}$. The active layer thickness is designed to be $40 \mathrm{~nm}$ in order to obtain enough spatial resolution to the standing wave. The absorbed power of the incident light is estimated to be about $0.4 \%{ }^{(9)}$. Inside such a thin Si film it is difficult to create a pn junction in the thickness direction, which is the structure of the conventional photodiode ${ }^{(10)}$. In the ultra-thin film photodiode, the $p n$ junction is designed in the lateral direction in the $\mathrm{Si}$ film. The depletion region grows laterally in the Si film.

(a)

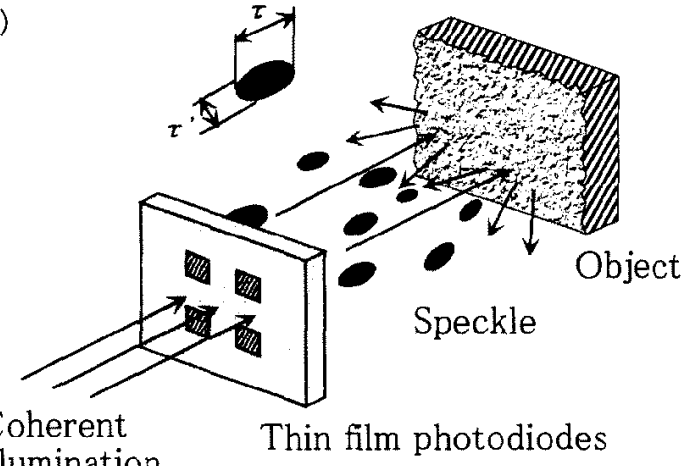

(b)

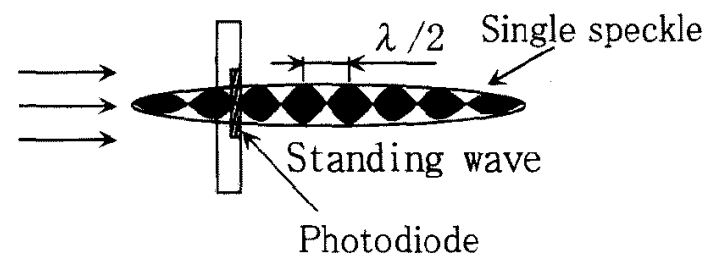

Fig. 2. Interferometer detecting the standing wave in a speckle's granular structure.

In many industrial applications to measure displacement, the surface of the object is optically rough and not a smooth mirror. The reflection light from a rough surface becomes speckle's granular structure including many different wavefronts. One of the wavefronts in the speckle will be parallel to the incoming light. By superposing it with the incoming laser beam, the standing wave will occur in these small granular spots. Figure 2 shows the principle for the second interferometer to detect the standing wave inside a speckle. This interferometer simply consists of an array of thin film photodiodes and a coherent laser light source as shown in Fig.2(a). The isolator is not necessary, since the back-scattered light from a rough surface does not couple into the laser source. The photodiode is again so thin as to allow an incoming light to pass through. The reflected light from the rough surface generate a speckle field. The speckle field interferes with the incident light and makes the standing waves in 
the bright speckles. The interference signal will be obtained in a bright speckle's granular structure. When a transparent thin film photodiode is placed in a bright granular field as illustrated in Fig.2(b), the displacement can be measured from the photodiode current. Although the phase of the interference fringe in the speckles is distorted randomly in a large area, the phase change can be measured monitoring the signal in the limited area of a single speckle. The interference signal will be weak and buried in a large offset. From the feature of standing waves, a modulation technique can be applied in a simple manner to oscillate the thin film photodiode. When the thin film photodiode is moved along the propagating direction of the laser beam, the signal phase changes. This feature is distinctive from the usual two-beam interferometer. In a usual two-beam interferometer the interference fringe is fixed to the propagating direction of the laser beam. Thus the small signal can be extracted from the large background.

When a bright speckle spot moves out and a dark spot overlaps with the photodiode area, the interference signal decreases. If only one photodiode is used, the probability for the photodiode to overlap with the bright speckles will be low. In order to increase this probability, the number of photodiodes is increased as shown in Fig.2(a).

When the roughness of the object is larger than the wavelength of the incident light, the statistical size of the speckle granular structure does not depend on the roughness of the surface. The length of the granular structure along the optical axis is given statistically by $\tau$ and the width is represented by $\tau$ ' in the diffraction field ${ }^{(11)}$.

$$
\begin{aligned}
\tau & =\frac{2 \lambda f^{2}}{a^{2}} \\
\tau^{\prime} & =\frac{3.8 \lambda f}{2 \pi a}
\end{aligned}
$$

Here, $\lambda$ is the wavelength of the incident light. $a$ is the radius of the laser beam on the object surface, and $f$ is the distance from the object. When the values of $a$ and $f$ to be around $0.5 \mathrm{~mm}$ and $30 \mathrm{~mm}$ respectively, $\tau$ is estimated to be $4.6 \mathrm{~mm}$ for wavelength of $632.8 \mathrm{~nm}$. This means that the displacement up to $\tau$ can be measured without changing the granular spot for detecting the standing wave in average. The value of $4.6 \mathrm{~mm}$ is a small length but may be long enough for a short-range measurement. The value of the width $\tau^{\prime}$, is calculated to be $23 \mu \mathrm{m}$. The size of the photodiode is designed as small as the transverse size $\tau$ ' of the speckle granular structure in order to avoid mixing some bright speckle spots by one photodiode.

\section{Fabrication and sensor package}

Figure 3 shows the fabrication sequence. The initial Si-on insulator wafer is prepared by direct wafer bonding between $\mathrm{Si}$ and quartz. The Si layer is thinned to $60 \mathrm{~nm}$. The comb-shaped windows are opened by lithography, through which As and B ions are implanted to make a $p n$ junction. After annealing and $\mathrm{SiO}_{2}$ deposition, etching to make the contact hole and $\mathrm{Al}$ electrode formation are carried out. Finally, the phase shifter is prepared by etching the deposited $\mathrm{SiO}_{2}$ layer. This final process can be omitted for the photodiodes detecting the standing waves inside speckles. Figure 4 shows the fabricated dual thin film photodiode having $4 \mu \mathrm{m}$ pitch comb shape. The $p^{+}$and $n^{+}$regions have the comb shape. The depletion region grows laterally between $p^{+}$and $n^{+}$regions. This design is to lengthen the depleted region and gather photocarriers as many as possible. The estimated thickness of the $\mathrm{Si}$ active layer is $35 \sim 40 \mathrm{~nm}$. The overall transmission rate reaches $70 \%$ in power, including the reflections at interfaces between $\mathrm{Si}$ and $\mathrm{SiO}_{2}$, and between $\mathrm{SiO}_{2}$ and air. Figure 5 shows typical current-bias voltage curves for the thin film photodiode, which is measured under illuminations of different incident power. The sensitivity is about $0.75 \mathrm{~mA} / \mathrm{W}$ and about 3 order smaller than that of the usual bulk Si photodiode. The measured series resistance and equivalent capacitance of the photodiode are $100 \mathrm{~K} \Omega$ and $15 \mathrm{pF}$ respectively. Due to the small cross-sectional area of the thin film photodiode, the series resistance is large whereas the parallel capacitance is small. The photodiode itself is considered to have the response speed upto $600 \mathrm{KHz}$. Figure 6 shows the fabricated photodiode array for detecting the standing waves inside a speckle field. There are four cell photodiodes. Each photodiode cell is $30 \mu \mathrm{m} \times 30 \mu \mathrm{m}$ in area.

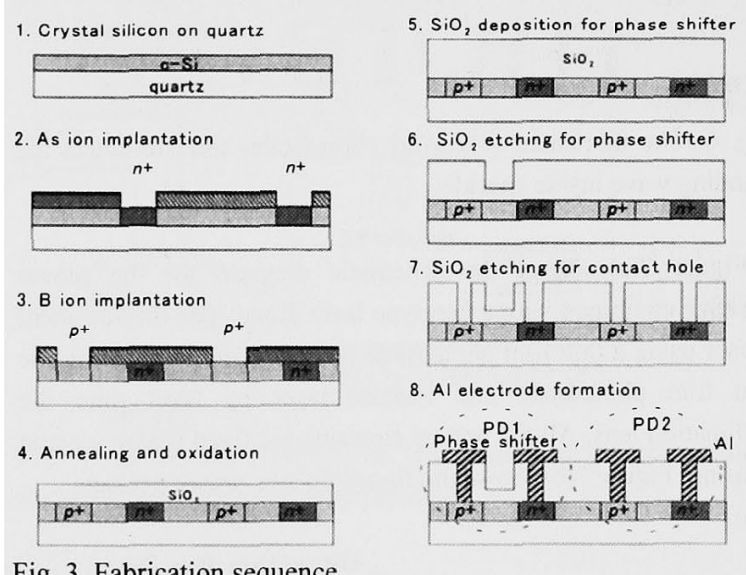

Fig. 3. Fabrication sequence

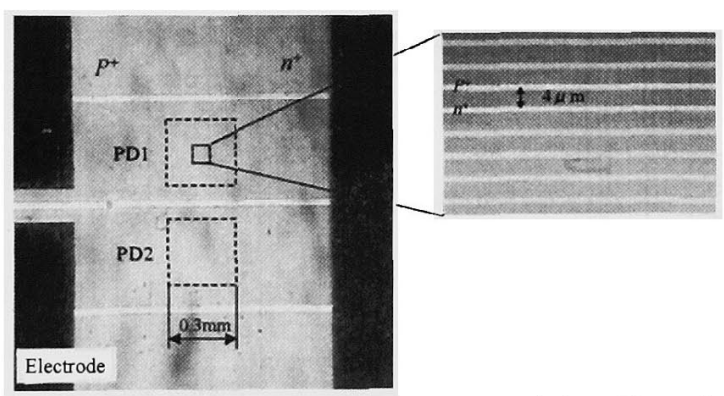

Fig. 4. The fabricated dual thin film photodiode with periodic electrode of $4 \mu \mathrm{m}$ pitch comb shape. 


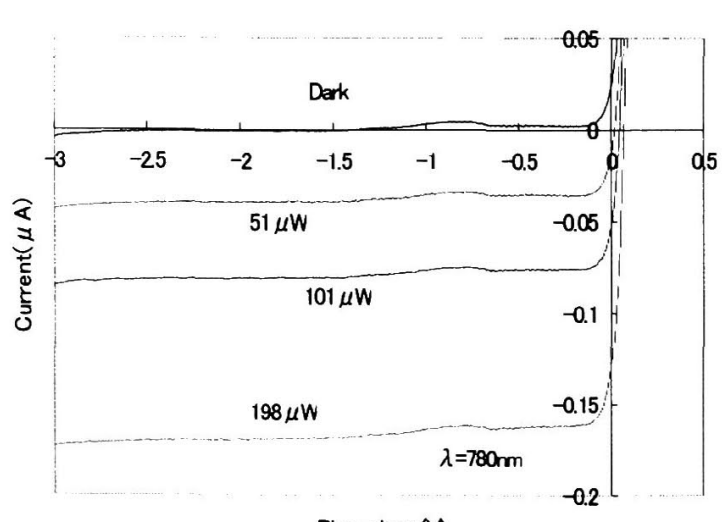

Bias voltage( $($ )

Fig. 5. Typical current-bias voltage curves of the thin film photodiode with periodic electrode of $4 \mu \mathrm{m}$ in pitch.

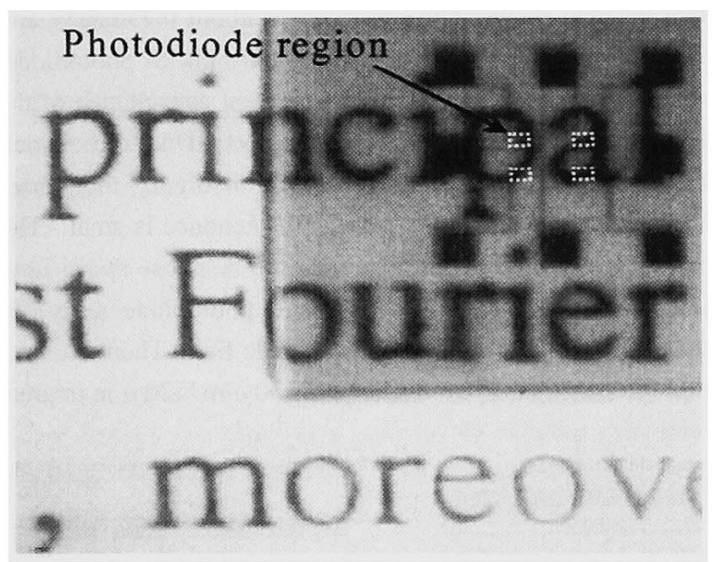

Fig. 6. The fabricated 4 arrayed photodiodes used to detect the standing wave inside speckle.

Figure 7(a) shows the schematic diagram for the sensor package combined with a can type laser diode. The displacement sensor using a thin film photodiode is packaged by stacking the thin film photodiode and isolator layer by layer onto the collimation lens. All the optical elements are fixed within a metal housing. Figure 7(b) shows the finger-tip size sensor package.

(a)

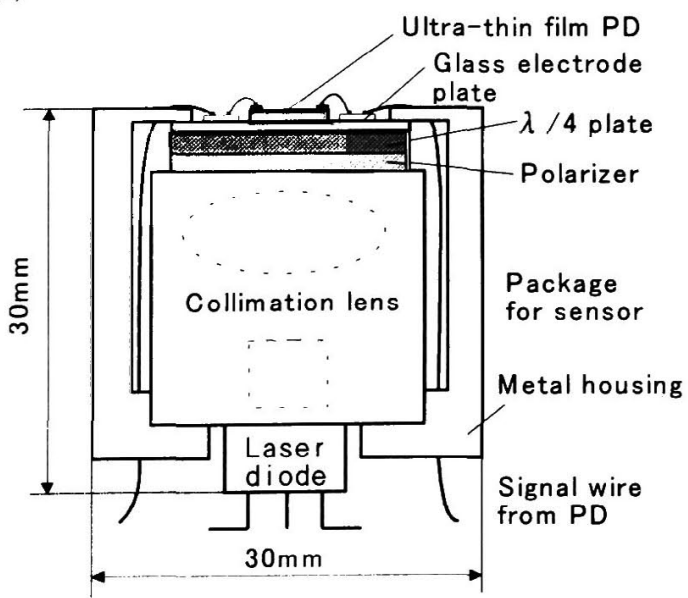

(b)

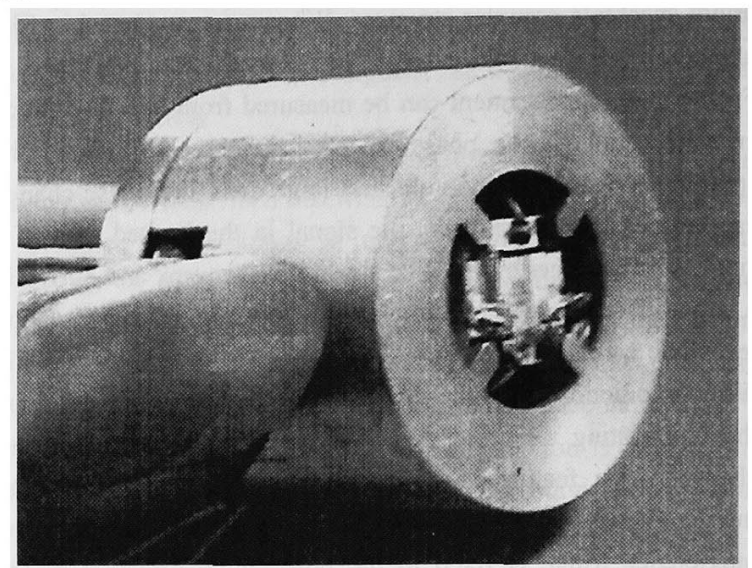

Fig. 7(a). Schematic diagram of sensor package. (b). Whole view of the packaged sensor.

\section{Experiments and results}

4.1 Detecting the standing wave generated by a flat mirror

The experiments are at first carried out with the bulk optical stages for aligning each element precisely. The experimental setup is same as that of Fig. 1(a). The red(632.8nm, $7 \mathrm{~mW}) \mathrm{He}-\mathrm{Ne}$ laser is used as the light source and a fabricated photodiode having $40 \mu \mathrm{m}$ pitch of $p^{+}$and $n^{+}$regions is used as the detector. The reflection mirror is placed $\sim 45 \mathrm{~mm}$ away from the thin film photodiode and moved by a piezo actuator. Figure 8 shows the obtained interference signal. The signal period agrees well with $\lambda / 2$. The signal amplitude over $1 \mu \mathrm{A}$ is obtained. The contrast reaches $77 \%$ at maximum, when the contrast is simply judged from the maximum and minimum signal magnitudes. The signal 1 of Fig. 8 is rather triangular. The interfaces in the thin film photodiode $\left(\mathrm{Si} / \mathrm{SiO}_{2}, \mathrm{SiO}_{2} /\right.$ air $)$ have significant reflection coefficient due to the difference in refractive indexes. At normal incidence on the thin film photodiode and the mirror, the FabryPerot effect occurs due to multiple reflections. When the thin film photodiode is a little slanted( $0.5 \mathrm{mrad})$ to the wavefront of the incident laser beam, the interference signal becomes sinusoidal as shown by signal 2 . In this case, the signal magnitude decreases.

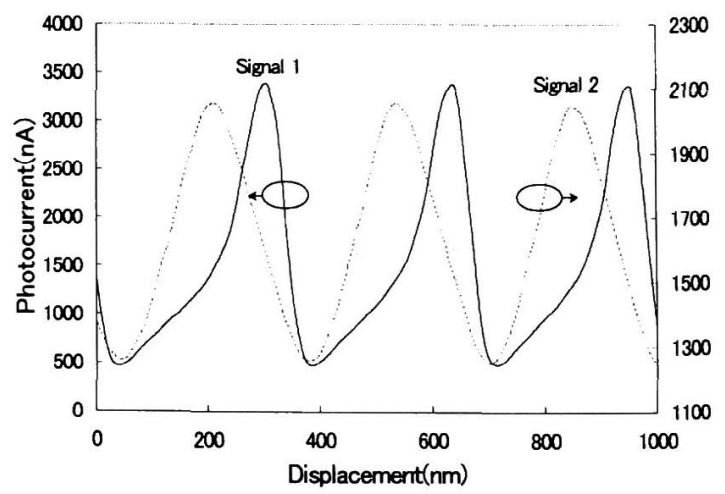

Fig. 8. Interference signal obtained from a thin film photodiode. 
Figure 9 shows two interference signals obtained from the dual thin film photodiode combined with a phase shifter. The optical setup is the same as that of Fig.1. The phase shift agrees well with the designed value of $\pi / 2$. Using this phase relation, the moving dircction of the mirror can be determined.

Figure 10 shows the interference signals obtained from the packaged sensor, in which a laser diode $(655.2 \mathrm{~nm}, 10 \mathrm{~mW})$ is used as the light source and the fabricated photodiode having $4 \mu \mathrm{m}$ pitch of $p^{+}$and $n^{+}$regions is used as the detector. The contrast reduces to $\sim 25 \%$. It mainly comes from the inaccurate alignment between the thin film photodiode and the laser beam. The planar components are just placed on the can type laser diode and fixed. The alignments between these components are determined by the flatness of the components.

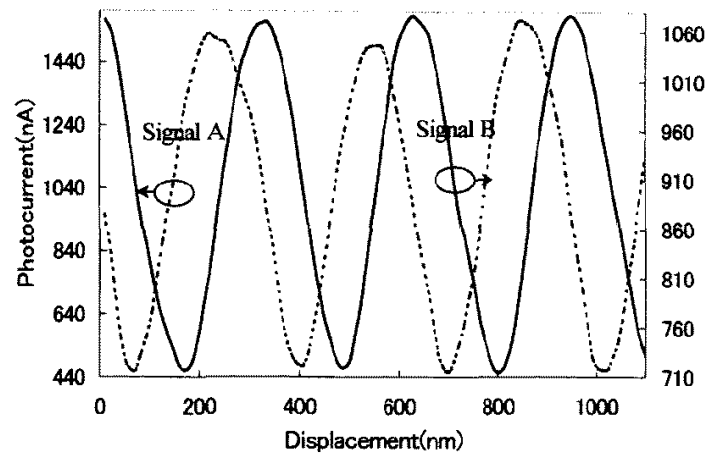

Fig. 9. Two interference signals obtained from the dual thin film photodiode combined with a phase shifter.

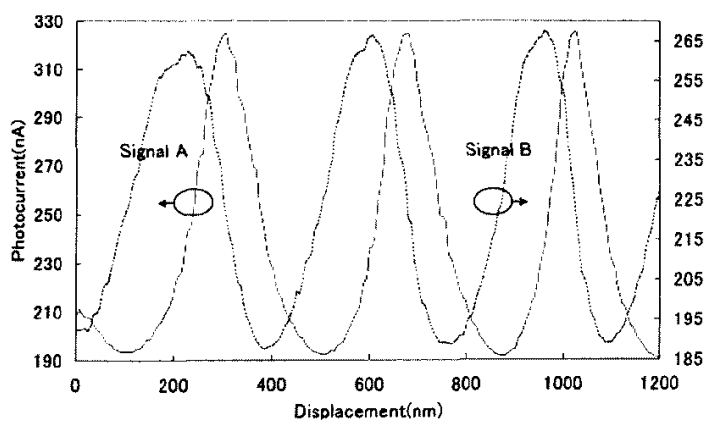

Fig. 10. The interference signal obtained from the packaged sensor.

\subsection{Detecting the standing wave inside a speckle field generated by a rough surface}

The standing wave inside a speckle field is measured using a rough sample surface. The experimental setup is shown in Fig. 11. The light source is a red $(632.8 \mathrm{~nm}, 7 \mathrm{~mW}) \mathrm{He}-\mathrm{Ne}$ laser. A sample object with the rough surface is placed about $30 \mathrm{~mm}$ away from the thin film photodiode. In the measurement, the thin film photodiode is sinusoidally oscillated by an actuator with an amplitude of $300 \mathrm{~nm}$ at a frequency of $300 \mathrm{~Hz}$. The sample object with the rough surface is slowly moved by $2 \mu \mathrm{m}$ with another actuator. The interference signal $\mathbf{S}(\mathrm{t})$ is inputted to an analogue phase detecting circuit in order to obtain the fundamental harmonic signal component $Y_{1 F}$ and the second harmonic component $Y_{2 F}$ respectively taking the oscillation voltage as the reference. The values of $Y_{1 F}$ and $Y_{2 F}$ are proportion to sine and cosine signal respectively. They change according to the phase change due to the sample displacement.

Figure 12 shows an example of the interference signal obtained directly from the photodiode as a function of the displacement. The period is in good agreement with $\lambda / 2$. The interference signal is $<10$ nA in peak-to-peak amplitude and $\sim 1 \%$ in contrast. The signal is sinusoidal in shape. The rough surface decreases the signal magnitude, on the other hand it makes the multiple reflections negligible and the alignment between the thin film photodiode and the object easy. Even when the object is slanted to the thin film photodiode, the signal can be obtained, since almost all wavefront directions are included in the speckle field. The interference signal can be selectively extracted from the large offset using the sinusoidal phase modulation technique ${ }^{(12)}$.

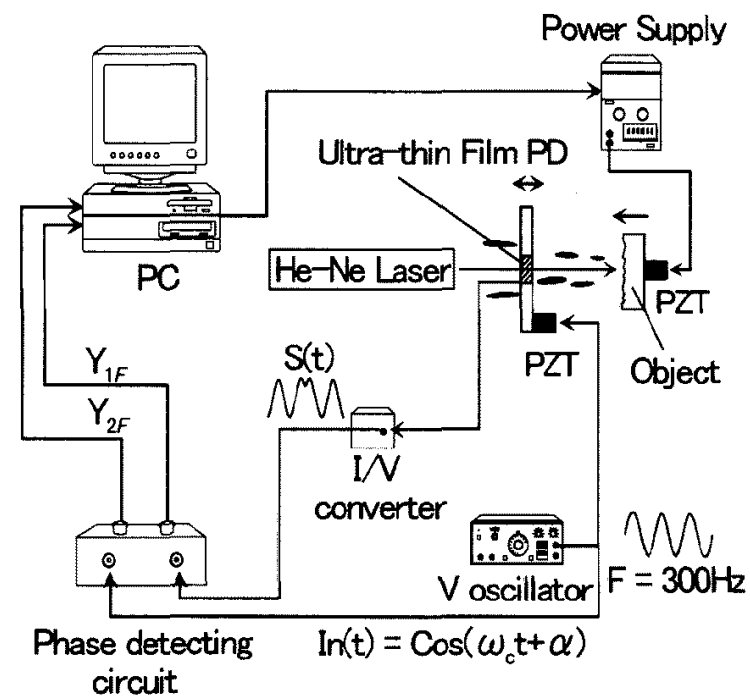

Fig. 11. Schematic diagram of the displacement measurement using a sinusoidal phase modulation.

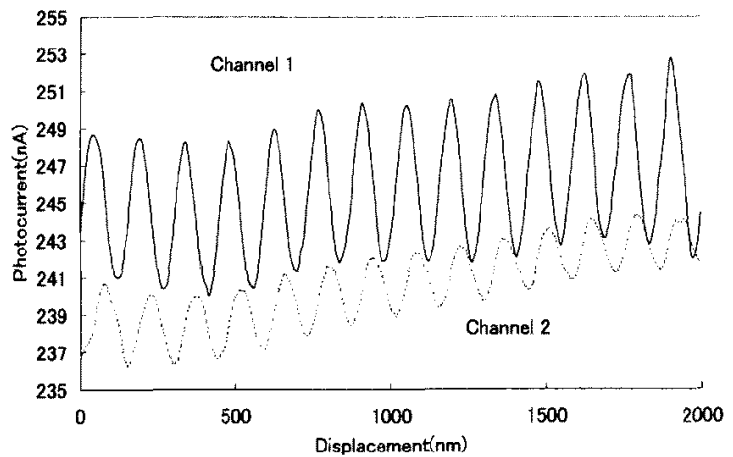

Fig. 12. Interference signals from photodiodes as a function of displacement. 
Output signals from the phase detecting circuit are shown in Fig.13(a), confirming the phase difference between two signals. Figure 13(b) shows the estimated displacement as a function of the voltage applied to the piezoelectric actuator. The measured values agree well with the expected linear motion obtained from the characteristics of the piezoelectric actuator. The displacement can be detected with a precision of $\pm 30 \mathrm{~nm}$ in the range of $2 \mu \mathrm{m}$. The actuator does not move at the region lower than $5 \mathrm{~V}$. This experimental curve is real and the performance of the used piezoelectric actuator. The displacement measurement is repeated at different positions from the object. The interference signal can be obtained at a probability of $50 \%$, corresponding to the ratio of the bright speckle region. By increasing the number of the photodiodes, this probability can approach toward $100 \%$.

(a)
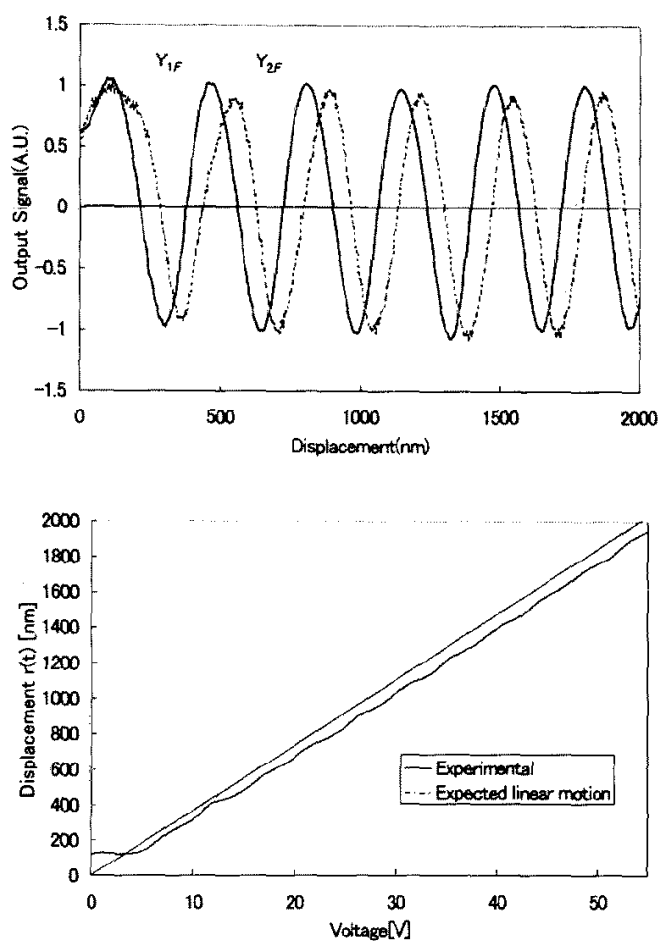

(b)

Fig. 13 (a). Signal $\mathrm{Y}_{1 F}$ and $\mathrm{Y}_{2 F}$ obtained from the phase detecting circuit as a function of the sample displacement. (b). Measured displacement as a function of the voltage applied to the piezoelectric actuator.

\section{Conclusions}

Two new interferometric displacement sensors are described. The sensors are suitable for the integration due to its simple optical setup. The principles of these interferometers are based on standing wave detection. This technique is compatible with the almost previously developed techniques. The ultra-thin film photodiode makes it possible to use optical standing waves in the metrological applications including the speckle field. The displacement direction can be measured, and the modulation can be applied not changing the optical setup significantly. The sensor package is constructed using a can type laser diode, an isolator (combination of a polarizer and a waveplate), and the ultra-thin film photodiode. The size is as small as finger tip.

\section{Acknowledgments}

We thank $O$. Sasaki of Niigata University for his useful discussions. A part of this work was performed in Venture Business Laboratory of Tohoku University.

(Manuscript received June 16,2000, revised November 24, 2000)

\section{References}

(1) K. J. Gasvik, Optical Metrology(John Wiley \& Sons, Ltd, West Sussex, England); P. Hariharan, Optical Interferometry (Academic, N. S. W., Australia).

(2) T. Suhara, T. Taniguchi, M. Uemukai, H. Nishihara, T. Hirata, S. Iio, and M. Suehiro, Monolithic interated-optic position/displacement sensor using waveguide gratings and QW-DFB laser, IEEE Photo. Technol. Lett.,7, 1195-1197, 1995.

(3) D. Hofstetter, H. P. Zappe, and R. Dandliker, Monolithically integrated optical displacement sensor in GaAs/AlGaAs, Electron. Lett., 31, 2121-2122, 1995.

(4) S. Merlo and S. Donati, Reconstruction of displacement waveforms with a single-channel laser-diode feedback interferometer, IEEE J. Quantum Electron. 33, 527-531, 1997.

(5) J. A. Smith, U. W. Rathe, and C. P. Burger, Laser with optical feedback as displacement sensors, Opt. Eng., 34, 2802-2810, 1995

(6) J. Kato, N. Kikuchi, I. Yamaguchi, and S. Ozono, Optical feedback displacement sensor using a laser diode and its performance improvement, Meas. Sci. Technol. 6, 45-52, 1995.

(7) M. Sasaki, X. Mi, and K. Hane, Standing wave detection and interferometer application using a photodiode thinner than optical wavelength, Appl. Phys. Lett., 75, 2008-2010, 1999.

(8) K. J. Gasvik, Optical Metrology (John Wiley \& Sons, Ltd., West Sussex, England), 1995, Chap. 8.

(9) S. M. Sze, Semiconductor Devices Physics and Technology (John Wiley \& Sons, Inc., New York, USA), 1985, 749-754.

(10) H. I. Liu, J. A. Burns, C. L. Keast, and P. W. Wyatt, Thin silicide development for fully-depleted SOI COMS technology, IEEE Trans. Electron Deveces, 45, 1099-1104, 1998.

(11) I. Yamaguchi, Fringe formations in deformation and vibration measurements using laser light, Progress in Optics XXII, E. Wolf (Elsevier Science Publishers B. V.), 282-294, 1985.

(12) T. Suzuki, O. Sasaki, S. Takayama and T. Murayama, Realtime displacement measurement using synchronous detection in a sinusoidal phase modulating interferometer, Opt. Eng. 32, 1033-1037, 1993. 
Xiaoyu Mi (Non-member) received the B.E. degree and the M.S.

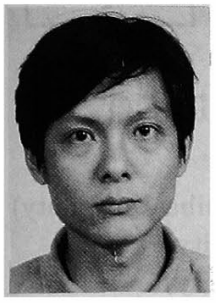
degree from Beijing University of Posts \& Telecommunications(China) in 1990, and Tohoku University in 1999 respectively. From 1990 to 1996, he had been with Wuhan Research Institute of Posts \& Telecommunications, Ministry of Posts \& Telecommunications, China. Since 1996, he has been with Hane Lab., Department of Mechatronics \& Precision Engineering, Tohoku University. Now he is a doctoral candidate there and engaged in research and development of integrated laser interferometer.

Minoru Sasaki (Member) received the M.S. and Dr. Eng. Degree

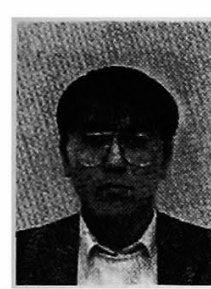
from Nagoya University in 1993 and 1995, respectively. In 1996, he was a Research Fellow of the Jap an Society for the Promotion of Science. From 1996 to 2000, he had been a research associate of the Department of Mechatronics and Precision Engineering, Tohoku University. Since 2000, he has been an assistant professor there and is currently engaged in the research and development of semiconductor micro-sensors. He received the Numata Memorial Award of Japan Society for Precision Engineering in 1994.

Takayuki Hirano (Non-member) received the B.S. and M.S.

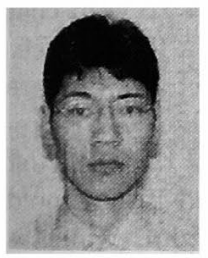
degree from Tohoku University in 1998 and 2000 , respectively. He was engaged in the research of optical sensor when he was a master course student of the Department of Mechatronics and Precision Engineering, Tohoku University. Since 2000, he has been with Toyada Automatic Loom Works, Ltd.

Kazuhiro Hane (Member) received the M.S and Dr. Eng. Degree

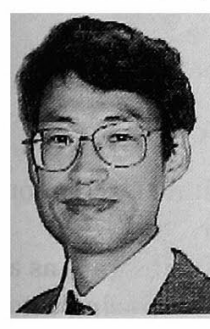

from Nagoya University in 1980 and 1983, respectively. From 1983 to 1994 , he worked as a member of the Dept. of Electrical Eng. in Nagoya University. From 1985 to 1986 , he was a guest researcher of National Research Council of Canada. Since 1994, he has been a professor in the Dept. of Mechatronics and Precision Eng., Tohoku University, and is currently engaged in the research and development of micro-sensors and optomechanical system. He was awarded the $17^{\text {th }}$ vacuum technology prize in 1992, the Numata Memorial Award of Japan Society for Precision Engineering in 1994, and the development Award of Japan Society for Precision Engineering in 1996, respectively. 